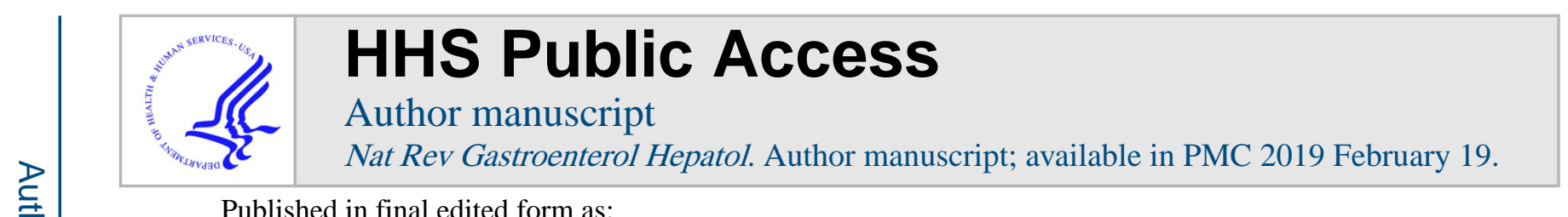

Published in final edited form as:

Nat Rev Gastroenterol Hepatol. 2017 August ; 14(8): 455-466. doi:10.1038/nrgastro.2017.71.

\title{
Extracellular vesicles in liver disease
}

Gyongyi Szabo and Fatemeh Momen-Heravi

Department of Medicine, University of Massachusetts Medical School, LRB208, 364 Plantation

Street, Worcester, MA 01605. USA.

\section{Abstract}

Extracellular vesicles (EVs) are membranous vesicles originating from different cells in the liver. The pathophysiological role of EVs is increasingly recognized in liver diseases including alcoholic liver disease, NAFLD, viral hepatitis and hepatocellular carcinoma. EVs, via their cargo, can provide communication between different cell types in the liver and between organs. EVs are explored as biomarkers of disease and could also represent therapeutic targets and vehicles for therapeutic delivery. Here, we review advances in understanding the role of EVs in liver diseases and discuss their utility in biomarker discovery and therapeutics.

\section{Blurb}

Extracellular vesicles have emerged as having key roles in liver disease. In this Review, Szabo and Momen-Heravi discuss the functions of extracellular vesicles in liver disease pathogenesis and progression, and explore their potential use as biomarkers, therapeutic targets and tools for therapy delivery.

The terminology referring to extracellular vesicles (EVs) has been changed substantially as understanding of EV biology has increased and the definitions of 'microvesicles', 'exosomes' and 'microparticles', that were used interchangeably in the past, have been redefined. ${ }^{1-5}$ Exosomes are homogeneous vesicles $(50-150 \mathrm{~nm})$ that originate from multivesicular bodies (MVB) and represent a discrete subpopulation of the greater family of $(\mathrm{EVs})^{2,6,7}$ (FIG. 1). On the basis of mode of biogenesis and surface protein expression, exosomes (50-150 nm) are differentiated from other types of extracellular vesicles such as microvesicles $\left(100-1000 \mathrm{~nm}\right.$ ) and apoptotic bodies $(500-2000 \mathrm{~nm}) .{ }^{8} \mathrm{In}$ contrast to exosomes, microvesicles are directly formed and released from plasma membrane. Several proteins, such as flotillin 1 , heat shock $70 \mathrm{kDa}$ proteins or major histocompatibility complex (MHC) class I and class II proteins, were historically used as exclusive exosomal subfraction biomarkers. However, new reports have suggested the presence of some of those markers in other subpopulations of extracellular vesicles. ${ }^{9}$ With the rapid advances in "omics' technology, specific molecular patterns will further our knowledge about different types of vesicles and improve classification. However, inconsistencies in the isolation

Correspondence to G.S. gyongyi.szabo@umassmed.edu.

Competing interests statement: the authors declare no competing interests

Further information

Extracellular RNA Communication Consortium: http://exrna.org

International Society for Extracellular Vesicles: https://isev.org 
methods and mixed methodology and terminology used in the past make the distinction of subtypes of those vesicles difficult. We will refer to both microvesicles and exosomes using the collective term 'extracellular vesicles'.

The cargo of EVs contains proteins, lipids and nucleic acid, coated with a lipid bilayer. EVs are secreted into the extracellular space, and thereby the circulation, by various cell types of the liver. ${ }^{10-12}$ (FIG. 2) The EV cargo demonstrates a snapshot of the parental cell at the time of release and can change depending on the stimulation status and/or differentiation stage of the cell. ${ }^{1314,15}$ This observation suggests that EV release and content are dynamic, providing opportunities and challenges in the evaluation of extracellular vesicles as biomarkers. EVs contain different macromolecules, including nucleic acids, proteins, and lipids. ${ }^{16-19}$ The composition of EVs might not be identical to the content of the cytosol of the cell of origin, suggesting active sorting of biomolecules into EVs. ${ }^{4}{ }^{15}$ In this Review, we focus on EVs and their role in physiology and the pathophysiology of liver disease and we will discuss their utility in biomarker discovery and their therapeutic potentials. Although EVs were considered initially as cellular by-products with insignificant biological importance, studies published in the past few years have identified EVs as conveyors of intercellular communication. ${ }^{16,20,21}$ Liver and other tissues shed EVs into the biological fluid and their abundance makes them compelling entities for a 'liquid biopsy', or a 'fluidome'.

\section{Liver as the source of EVs}

Although the detection of EVs production in vivo at the tissue level awaits development of new technologies, the in vivo presence of circulating EVs indicates that they are produced in specific tissues and cells. ${ }^{22}$ Most cell types in the liver have been shown to produce EVs under in vitro conditions, including hepatocytes, cholangiocytes, hepatic stellate cells (HSCs), sinusoidal endothelial cells (SECs), Kupffer cells and other different immune cell populations (FIG. 3) ${ }^{23-29}$ Hepatocytes, including primary murine and human hepatocytes as well as hepatoma cell lines secrete exosomes and microvesicles, and their production can change quantitatively and qualitatively in response to cellular stimulation and under different disease conditions. ${ }^{16,30}$ Proteomic analysis revealed the presence of $\sim 251$ proteins in EVs derived from primary rat hepatocytes. ${ }^{31}$ In addition to transmembrane proteins, such as tetraspanins described in EVs from other cell types (including CD63, CD81 and CD82) and the asialoglycoprotein receptor (ASGR), enrichment of hepatocyte-specific receptors was also found in hepatocyte-derived EVs. ${ }^{31}$ The hepatocyte-derived EV cargo included proteins involved in the endosomal pathway and detoxification, cytosolic proteins including cytochromes, as well as secreted proteins such as coagulation-related proteins and apolipoproteins. ${ }^{31}$ These findings suggest that the protein and nucleic acid composition of cell-derived EVs can change in response to cellular stress or other stimulations, raising the question of whether EVs released under cell or organ stress and disease conditions could have a different biological role compared with normal homeostasis or could serve as biomarkers. ${ }^{16,31}$

Biliary microvesicles and exosomes were found to attach to rat cholangiocyte cilia, and resulted in a decrease in the ratio of phosphorylated extracellular signal-regulated kinases 
(ERK) $1 / 2$ to total ERK $1 / 2$, and increased miR-15A expression and reduced cholangiocyte proliferation. ${ }^{32}$ Biliary EVs in chicken enhanced $\mathrm{CD}^{+}{ }^{+}$and $\mathrm{CD} 8+\mathrm{T}$ cell proliferation and macrophage activation in the liver and and inhibited avian leukosis virus replication in DF-1 cell line. ${ }^{33}$

Circulating levels of leuko-endothelial $\left(\mathrm{CD} 31^{+} \mathrm{CD} 41^{-}\right)$, pan-leukocyte $\left(\mathrm{CD} 11 \mathrm{a}^{+}\right)$, lymphocyte $\left(\mathrm{CD}^{+}\right)$and erythrocyte $\left(\mathrm{CD} 235 \mathrm{a}^{+}\right) \mathrm{EVs}$ were found to be increased in patients with cirrhosis compared with healthy controls. In patients with cirrhosis hepatocyte-derived EVs in the plasma contained elevated levels of cytokeratin-18 that was not present in healthy subjects. The severity of cirrhosis was found to be correlated with the levels of leukoendothelial and hepatocyte derived EVs. Those EVs had functional effects, as they impaired contraction of vessels in response to phenylephrine in a manner dependent on prostaglandin $\mathrm{G} / \mathrm{H}$ synthase 1 (also known as cyclooxygenase 1) in rat aortic rings. ${ }^{34} \mathrm{EVs}$ (enriched in the exosome fraction) derived from SECs were found to modulate HSC function. Internalization of SEC-derived EVs triggered sphingosine-1-phosphate-dependent migration of HSCs, demonstrating that EVs mediate communication between sinusoidal endothelial cells and stellate cells. ${ }^{35}$

The rich repertoire of immune cell populations in the liver provides another important source of EVs. Virtually all immune cell types including T and B lymphocytes, myeloid and plasmacytoid dendritic cells, macrophages, Kupffer cells and neutrophil leukocytes produce EVs. ${ }^{36-39}$ In vitro studies indicate that immune cell-derived EVs provide communication between the different cell types in the liver, both within the immune cell compartment and between immune and parenchymal cells. However, the full functional potential of liver and hepatocyte-derived EVs is yet to be fully understood. Hepatocyte-derived EVs (and not EVs from other liver cell types) were shown to mediate liver repair and regeneration by transferring ceramidase and sphingosine kinase 2 that increases synthesis of sphingosine-1phosphate production in target hepatocytes in ischaemia-reperfusion injury or after partial hepatectomy. ${ }^{40}$ In another study, EVs enriched with microRNA (miR)-122 derived from hepatocytes after alcohol exposure were taken up by macrophages, resulting in transfer of hepatocyte-derived miR-122 (FIG. 4). ${ }^{16}$ Although unstimulated macrophages have undetectable levels of miR-122, miR-122 transferred by EVs sensitized macrophages to increased TNF production in response to lipopolysaccharide (LPS) stimulation. ${ }^{16}$ Macrophage-to-macrophage communication was also found via exosomal transfer of miR-27a after alcohol exposure, resulting in preferential induction of the M2, or alternatively activated, macrophage phenotype. ${ }^{14}$ Thus, these findings demonstrate that EVs released by one cell type are taken up by other cells and thereby mediate transfer of biomolecules, such as miRNAs, that alter functions of recipient cells. These observations open a new paradigm in intercellular communication in the liver, regulated by EVs.

\section{Liver as the target of EVs}

At this time the biogenesis and biodistribution of EVs is only partially understood (Box 1). That EVs can interact with different cells in the liver through specific receptors and cellular uptake is already known. For example, EVs derived from hepatocytes can modulate function and activation of liver macrophages or mediate liver regeneration. ${ }^{4}$ Specific EV ligands, 
such as tetraspanins or and major histocompatibility complex (MHC)I and II, can interact with the target cells by different signalling pathways. ${ }^{41} \mathrm{EV}$ uptake by most cell types is via endocytosis, including clathrin-dependent endocytosis and clathrin-independent pathways, such as caveolin-mediated uptake, phagocytosis, macropinocytosis and lipid-raft-mediated internalization. ${ }^{42}$ Lipid and protein interactions facilitate EV uptake, and tetraspannins, integrins, immunoglobulins, peptidoglycans and lectins all contribute to EV uptake by target cells. $^{42}$

Several studies have demonstrated that the liver is an active site of EV uptake. Imai et al. showed that PKH26-labelled exosomes derived from the mouse melanoma cell line B16BL6 were taken up by macrophages in the liver and spleen, but not in the lung, after intravenous administration. ${ }^{43}$ Bala et al. showed that after intravenous administration of miR-155enriched exosome-rich plasma into miR-155-deficient mice, most of the miR-155 was found in the liver with a lower level in the kidney. ${ }^{44}, 45$ This study suggests rapid biodistribution and uptake of EVs in vivo, with maximal serum levels at 5 minutes after injection and no detectable circulating levels after $30 \mathrm{~min} .{ }^{44,45}$ Cellular uptake of EV-transferred miR-155 was found both in hepatocytes and in liver mononuclear cells, indicating rapid cellular uptake and clearance of circulating EVs by the liver. ${ }^{44,} 45$ The functional capacity of EVsderived miR-155 was indicated by increased pro-inflammatory cytokine induction in miR-155 knockout mice after exosomal transfer of miR-155. ${ }^{44,}{ }^{45}$ Exosome-derived miR-146a and miR-155 were shown in another study to modulate inflammatory responses to endotoxin. $^{20}$

Hepatocyte-derived EVs can also mediate liver repair and regeneration in ischaemiareperfusion injury and after partial hepatectomy. ${ }^{40}$ Interestingly, EVs derived from hepatocytes, and not other liver cell types, induced a dose-dependent proliferation of hepatocytes in vitro. Hepatocyte EVs taken up by target hepatocytes transferred neutral ceramidase and sphingosine kinase 2, causing increased synthesis of sphingosine-1phosphate in this model. Consistent with this mechanistic explanation, ablation of exosomal SK prevented the proliferative effect of exosomes. ${ }^{40}$

\section{EVs in viral hepatitis}

Studies from the past few years have shown that the exosome-enriched fraction of EVs can regulate $\mathrm{HCV}$ infection. HCV-infected human hepatocytes and Huh-7.5.1 cells produce EVs containing HCV RNA that complexes with miR-122, argonaute 2 and heat shock protein $90 .{ }^{46}$ Furthermore, these EVs can transfer HCV infection to non-infected hepatoma cells or primary human hepatocytes and trigger viral replication in these newly infected cells. ${ }^{46}$ Patients with chronic HCV infection have circulating serum EVs containing HCV RNA in single-strand or double-strand form, and are therefore able to mediate $\mathrm{HCV}$ infection to naive human hepatocytes. ${ }^{46,47}$ Although new therapies for $\mathrm{HCV}$ eradication are increasingly efficacious, exosome-mediated HCV infection is considered an alternative route of $\mathrm{HCV}$ transmission that can have an effect on treatment with oral antiviral therapies. ${ }^{46}$

Extracellular vesicles from cell culture supernatant of cells infected with GBV-C or serum from individuals with GB virus $\mathrm{C}(\mathrm{GBV}-\mathrm{C})$ infection, a human virus of the Flaviviridae 
family related to $\mathrm{HCV}$, were shown to inhibit $\mathrm{T}$ cell receptor signalling that was associated with the GBV-C envelope 2 protein found in EVs. ${ }^{48}$ The GBV found in the EVs was functional and transmitted viral RNA to peripheral blood mononuclear cell (PBMCs) in vitro resulting in productive infection. ${ }^{49}$ These observations highlight the importance of EVs in viral hepatitis infection and transmission.

Patients with chronic hepatitis $\mathrm{C}$ virus infection showed increased platelet activation and a percentage of platelet-derived EVs in the circulation compared to patients with chornic hepatitis B infection. The percentage of platelet-derived EVs in these individuals correlated with levels of liver fibrosis markers, such as serum hyaluronate and $\mathrm{N}$-terminal propeptide of type III procollagen, indicating the possible role of platelet activation, increased number of EVs and liver fibrosis. This may indicate a possible role for platelet activation increasing EV number and mediating liver fibrosis, a concept that deserves further investigation. ${ }^{50}$

In a 2013 study, Li et al. ${ }^{51}$ reported that in vitro IFNa treatment induced the transfer of HBV resistance from non-permissive liver nonparenchymal cells to permissive hepatocytes via EVs containing antiviral proteins. ${ }^{51} \mathrm{HBV}$ nucleic acids and proteins have also been found in EVs in the sera of patients with chronic HBV infection. ${ }^{52}$ These EVs interacted with natural killer cells and impaired their function, resulting in decreased IFN $\gamma$ production and reduced expression of ATP-dependent RNA helicase DDX58 (also known as retinoic acid-inducible gene 1 protein; RIG-I), an intracellular viral sensor receptor. In HCV infection, $\mathrm{HCV}$ induced monocyte differentiation into M2 macrophages, which in turn promoted HSC activation; this stellate cell activation effect was reproduced by EVs derived from HCV-infected hepatocytes. ${ }^{53}$ Collectively, these results suggest that EVs have an important role in viral transmission of both $\mathrm{HCV}$ and $\mathrm{HBV}$, as well as in undermining innate immune responses in chronic viral hepatitis.

\section{EVs in steatohepatitis}

In experimental steatohepatitis induced in mice by choline-deficient, L-amino acid-defined (CDAA) and high fat diets, the levels of circulating extracellular vesicles, both exosomes and microvesicles, were increased compared with controls. ${ }^{54}$ Proteomic analysis of the EVs revealed differences in the cargo between NAFLD and controls. The authors proposed that the liver was a source of the EVs on the basis of their miR-122 and miR-192 content because these are liver-predominant miRs abundantly expressed in hepatocytes. ${ }^{54}$ Lipotoxic activation of hepatocytes with free fatty acids was also shown to induce release of extracellular vesicles enriched in ceramide, and these vesicles induced macrophages chemotaxis, suggesting a mechanism for NASH-related inflammation. ${ }^{55}$ Consistently, highfat diet in rats increased the number of circulating EVs that promoted inflammation. ${ }^{56}$. Increased quantities of hepatocyte-derived EVs were detected in the blood of mice with high-fat-diet-induced steatohepatitis, and the increase in circulating extracellular vesicles correlated with disease severity. Those vesicles were internalized into HUVEC cells in vitro by a mechanism dependent on pantetheinase (also known as Vanin-1), and promoted angiogenesis and induced liver damage in steatohepatitis. Hepatocyte-conditioned media in which the EVs were removed by ultracentrifugation lacked proangiogenic activity ${ }^{57}$. In another report, patients with NAFL showed increased numbers of EVs originating from 
invariant natural killer $\mathrm{T}$ cells and $\mathrm{CD} 14^{+}$macrophages and monocytes, which have a pathogenic role in disease progression. The number of EVs correlated well with plasma levels of alanine transaminase (ALT) and the severity of disease as assessed by histology. ${ }^{58}$ In the context of NASH, it has been shown that lipids induce release of EVs from primary human and mouse hepatocytes through a death receptor 5 (also known as tumor necrosis factor receptor superfamily member 10B) signalling pathway, which then activate an inflammatory phenotype in macrophages. ${ }^{59}$ Consistent with this, mixed lineage kinase 3 (MLK3, also known as mitogen-activated protein kinase kinase kinase 11) induces the release of C-X-C motif chemokine 10 (CXCL10)-laden EVs from hepatocytes treated with lipotoxic lipids, which induce macrophage chemotaxis. MLK3-deficient mice fed a NASHinducing diet showed diminished number of total plasma EVs and EVs containing CXCL10 compared with wild-type mice. ${ }^{60}$

In patients with alcoholic hepatitis, the number of circulating EVs, particularly exosomes, was found to be higher compared with healthy individuals. ${ }^{16,61}$ Likewise, in a mouse model of alcohol-induced steatohepatitis, the number of circulating EVs was increased compared with the pair-fed controls, and these EVs were enriched in miR-122, miR-192 and miR-30. ${ }^{62}$ The clinical relevance of these findings was confirmed in patients with alcoholic hepatitis, in which increased numbers of circulating EVs containing elevated levels of miR-122, miR-192 and miR-309 were found. ${ }^{62}$ Acute alcohol binge drinking also increased the number of circulating EVs in healthy volunteers, and these EVs were enriched in miR-122 an miR-155. ${ }^{16}$ In vitro, alcohol treatment induced the release of EVs in hepatoma cells and in primary mouse and human hepatocytes. ${ }^{16}$ Alcohol treatment also induced EV release in macrophages, suggesting that EV production in alcoholic liver disease occurs in both parenchymal and immune cells. ${ }^{14}$ In alcoholic liver disease, hepatocyte-derived EVs enriched in miR-122 are taken up by macrophages, resulting in functional changes and sensitization of these cells to LPS-induced pro-inflammatory responses. ${ }^{16}$ These observation suggest an important and unexpected role for hepatocyte-derived EVs in modulation of inflammation in alcoholic diver disease (FIG. 4). A 2016 study by Verma et al., showed that hepatocytes release CD40-ligand -containing EVs in a caspase-dependent manner in response to alcohol exposure, and that these EVs promote macrophage activation and contribute to the inflammatory profile of alcoholic hepatitis. ${ }^{61}$ Together these observations suggest dynamic, EV-mediated communication between hepatocytes and macrophages in the liver.

\section{EVs in drug-induced liver injury}

Circulating extracellular vesicles are now in focus as potential biomarkers in DILI. ${ }^{63}$ In a mouse model of paracetamol-induced liver injury, increased serum miR-122 levels were found both in the exosomal rich and protein-rich serum fractions. ${ }^{64}$ In a rat model of paracetamol -induced DILI, increased numbers of circulating EVs were found and these EVs showed elevated levels of miR-122 and albumin mRNA. ${ }^{63}$ Increased serum miR-122 levels were also found early in patients with paracetamol-related liver injury and the microRNA profile in paracetamol-induced DILI showed some specificity compared with ischaemic hepatitis. ${ }^{65}$ In another study, proteomic analysis of EVs isolated from the sera of rats after D-galactosamine (GalN) or LPS administration showed a characteristic protein composition 
that correlated with liver toxicity, indicating the potential of EV protein cargo as a biomarker in DILI. ${ }^{24}$

\section{EVs in liver fibrosis}

The hallmark of liver fibrosis is activation of HSCs. A study by Chen et al. showed that quiescent HSCs produce EVs containing high levels of Twist related protein 1, which drives miR-214 expression and results in decrease in mRNA levels for connective tissue growth factor (CCN2). ${ }^{26}$ Exosomal Twist1 was shown to shuttle between activated and quiescent HSCs, and hepatocyte-derived EVs also regulated miR-214 expression in HSCs, demonstrating a complex intercellular interaction involving EVs and miRNAs in liver fibrosis. ${ }^{26}$ In another study, EVs derived from endothelial cells were internalized by HSCs, resulting in sphingosine-1-phosphate-dependent HSC activation and promotion of liver fibrosis. $^{35}$

\section{EVs in liver cancer}

Cancer cells, including hepatocellular cancer (HCC) cell lines, secrete increased numbers of EVs compared with non-cancer cells, and these EVs have pro-metastatic effects. ${ }^{66,67} \mathrm{EV}$ release from HCC cell lines was also increased after anticancer drug exposure and those EVs could activate natural killer cells to elicit antitumour immunity. ${ }^{68}$ This study also found that EVs containing heat-shock proteins (hsp60, hsp70, hsp90) stimulate cytotoxicity and granzyme B production in natural killer cells. ${ }^{68}$ Furthermore, stem cell-derived EVs (enriched for the microvesicle fraction) inhibited HCC growth and survival in a mouse model of the disease. ${ }^{28}$ Growth of HepG2 hepatoma cells was inhibited both in vitro and in vivo by addition of EVs microvesicles derived from human bone marrow mesenchymal stem cells (MSCs); however, the mechanism by which MSC-derived extracellular vesicles exert this effect is yet to be understood. ${ }^{69}$

EV-associated RNA from colorectal cancer cells is delivered to hepatoma cells and lung cell lines in vitro, and this observation has raised the possibility of cancer metastasis via exosome-mediated nucleic acid delivery. ${ }^{70} \mathrm{EVs}$ derived from pancreatic cancer promote liver metastasis by initiating pre-metastatic niche formation that involves uptake of exosome-derived factors, such as Macrophage migration inhibitory factor (MIF), causing Kupffer cell and hepatic stellate cell activation. This process generated a fibrotic microenvironment with immune cell infiltration that promoted metastasis. ${ }^{71}$ In a 2015 article by Wang et al., EVs derived from a highly liver metastatic colorectal cancer cell line (HT-29) increased the metastatic tumour burden and extension in the mouse liver of Caco-2 colorectal cancer cells, which usually demonstrate poor liver metastatic potential. It has been suggested by Wang et al that HT-29 cancer cells promote colorectal cancer metastasis by recruiting $\mathrm{C}-\mathrm{X}-\mathrm{C}$ chemokine receptor type 4 (CXCR4)-expressing stromal cells, which are permissive to metastatic niche formation. ${ }^{72}$ 


\section{EVs as novel biomarkers}

Biomarker discovery represents an approach for the early detection, monitoring and evaluation of treatment response in various liver diseases. Different definitions of biomarkers have been reviewed in Box 2. Although the gold standard approach is still liver biopsy, there is great demand for non-invasive markers of liver diseases, such as biomarkers and 'liquid biopsy' that could mitigate the need for the invasive liver biopsy. ${ }^{62,73,74}$. Currently, the blood-based assessment of liver injury extent is based on levels of hepatic enzymes including ALT, aspartate aminotransferase (AST), alkaline phosphatase and $\boldsymbol{\gamma}$ glutamyltranspeptidase (GGT). However, serum hepatic enzymes activities are not specific within a spectrum of different liver diseases and these traditional markers do not always correlate with the stage of liver disease or extent of hepatocellular injury and/or inflammation. ${ }^{75}$

Several studies documented the potential utility of EV-based biomarkers in liver diseases, especially in HCC. ${ }^{12,76-78}$ Using EVs as biomarkers potentially provides new dimensions in nucleic-acid based and protein-based diagnostics. First, due to membrane coating, EVs protect their protein and nucleic acid cargo from degradation, ${ }^{79}$ an attractive property for use in diagnosis. Second, EVs are enriched with highly selected markers during exosomal sorting, which otherwise constitute only a very small proportion $(<0.01 \%)$ of the total proteome of body fluids. ${ }^{80}$ This enrichment of diagnostic molecules in EVs facilitates identification of low abundance nucleic acid or protein biomarkers that normally would go undetected. In circulating EVs in healthy individuals, $\sim 66$ proteins were identified, most of which were involved in vesicular trafficking pathways. ${ }^{19,81}$ Third, confining biomarker discovery to EVs, as opposed to total plasma or serum, will decrease the complexity of the assay. ${ }^{24}$ Fourth, the number of EVs reflects the metabolism of cells in tissues and analysis of their cargo might provide direct information on disease progression, recovery and drug response. As such, the discovery of novel candidate nucleic acids and protein biomarkers in EVs could open new avenues of investigation in assessing progression/recovery/therapy response. Specific protein contents or nucleic acid contents of EVs have been suggested as biomarkers for detection of different liver disease (Table 1), yet none of the suggested biomarkers has been validated in independent studies, and none of the studies of these biomarkers in liver disease has adequately established sensitivity and specificity.

\section{EV proteins as potential biomarkers of liver disease}

Several potential protein-based EV biomarkers have been introduced for liver diseases (Table 1). Full length soluble receptor-type tyrosine-protein phosphatase $\gamma$ (sPTPRG) isoforms associated with EVs have been identified as a biomarker for liver injury in human and mouse plasma. ${ }^{82}$ A positive correlation between high plasma levels of sPTPRG and liver damage was observed in human plasma samples with low (ALT $<40 \mathrm{U} / \mathrm{ml}$ ) or increase $\left(\mathrm{ALT}>450 \mathrm{u} / \mathrm{ml}\right.$ ) levels. ${ }^{82} \mathrm{EVs}$ were also identified as conveyors of CTGF as they transferred CTGF between HSCs and subsequently amplified fibrogenic signalling. This finding suggests that EVs containing CTGF could have utility as a noninvasive biomarker to assess hepatic fibrosis. ${ }^{83}$ In another study, soluble CD81 was increased in serum EV fraction in patients with chronic hepatitis $\mathrm{C}$ compared with healthy subjects and patients who had 
recovered, and EV-associated soluble CD81 levels were associated with plasma ALT levels and severe forms of liver fibrosis. ${ }^{84}$

In a proteomic study on EVs derived from cultured hepatocytes and EVs from the sera of liver-injured mice, increased levels of some liver-specific proteins such as the enzymes carbamoyl phosphate synthase 1 (CPS1), $S$-adenosylmethionine synthase 1 (MAT), and catechol $O$-methyltransferase (COMT) were observed. ${ }^{24}$ In agreement with these results, upregulation of MAT enzyme levels have been previously shown to be required for the proper liver regeneration in hepatectomized mice. ${ }^{24,85}$ Circulating EVs enriched in liverorigin proteins were isolated from the plasma of patients with HCC using affinity isolation specific to the monoclonal antibody hepatocyte paraffin 1 (Hep Par 1). ${ }^{86}$ The levels of these liver-specific circulating EVs were increased in patients with HCC and correlate well with the size of the liver tumours. ${ }^{86}$ In another study on urine samples in an acute liver injury animal model, a set of differentially expressed proteins including CD26, CD81, SLC3A1 and CD10 were identified and proposed as biomarkers of liver disease. Notably, Dgalactosamine-treatment that incudes acute liver injury, results in a severe reduction in some proteins that normally are clearly detected in urinary vesicles in rats suggesting that protein composition of urinary EVs can reflect liver damage. ${ }^{87}$

\section{Nucleic acid cargo of EVs as biomarkers in liver diseases}

The cargo of EVs includes both proteins and nucleic acid, however, most studies to date focused on characterizing nucleic acid composition. EV-associated nucleic acids, especially microRNAs, have generated interest as biomarkers for various diseases (Table 1). Several microRNAs have been introduced as potential biomarkers of HCC. miR-718 showed decreased expression in serum EVs of patients with HCC with recurrence after liver transplant compared with those without recurrence. Decreased levels of miR-718 were associated with HCC tumour aggressiveness in a different cohort of patients. ${ }^{66}$ In another study, miRNA-21 was shown to be elevated in the serum EV fraction of patients with HCC compared with the EV-depleted serum fraction, and the level of serum exosomal miR-21 was higher in patients with HCC than in healthy controls and patients with chronic hepatitis B. High levels of emiR-21 expression were correlated with cirrhosis and advanced tumour stage. EV-associated miR-21 was reported to have higher diagnostic sensitivity and accuracy than miR-21 in whole serum. ${ }^{88}$

In a 2012 study, our group showed that increased serum and plasma levels of miR-122 correlated with ALT increases in different liver damage models induced by alcohol, paracetamol or TLR9 ligands (CpG dinucleotides and LPS). ${ }^{64}$ Both miR-155 and miR-146a, referred to as "inflammamiRs" 89 , were increased in the serum and plasma of mice with inflammation-related liver diseases such as alcohol-induced liver injury or after $\mathrm{CpG}$ and LPS administration. ${ }^{64}$ Both in alcoholic and TLR9- plus TLR4-mediated inflammatory liver injury models, serum and plasma miR-122 and miR-155 were predominantly associated with the EV-rich fraction in mice, in contrast to DILI in which these miRs were enriched in the protein-rich fraction. ${ }^{64}$ The number of circulating EVs was also increased in patients with acute alcoholic hepatitis compared with healthy controls. ${ }^{16}$ 
Increased numbers of EVs and elevated levels of miR-122 were also found in EVs isolated from the sera of patients with alcoholic hepatitis or healthy individuals after binge drinking alcohol, compared with healthy controls and individuals own established baseline levels, respectively. ${ }^{16,62}$ In addition to miR-122, levels of miR-192 and miR-30a were increased in EVs isolated from the sera of mice chronically fed ethanol or patients with alcoholic hepatitis. ${ }^{62}$ miR-192 showed the highest diagnostic accuracy of alcoholic hepatitis, identified by an area under the curve (AUC) of $0.96(P<0.001)$. miRNA-122 and miRNA-30a demonstrated an AUC of $0.92(P<0.001)$ and $0.85(P<0.05)$, respectively. ${ }^{62}$

\section{Discovery of EV biomarkers}

Despite EVs holding promise for new biomarker discovery, large studies on the diagnostic, prognostic and predictive value of EV biomarkers in different liver diseases are lacking (Box 1). Although EVs have been shown to associate with known markers of pathogenesis, association of EVs with patient-oriented outcomes such as prognosis and response to the treatment are yet to be evaluated. Moreover, independent comprehensive studies in wellcharacterized patient populations are needed to assess the sensitivity and specificity of EV biomarkers in relation to clinical outcomes. These studies will aid discoveries of EV biomarkers in liver diseases that should not be limited to miRNAs, and will probably involve other nucleic acids (for example long non-coding RNAs (lncRNAs)), proteins and lipid markers. Additional limitations include the requirement to standardize EV, exosome and microvesicle isolation procedures from various biofluids. Although there has been substantial progress in this area in the past 5 years through the NIH extracellular RNA Communication Consortium and the International Society for Extracellular Vesicles, challenges regarding isolation, pre-analytical and post-analytical aspects of EV biomarker use still remain. Although different isolation techniques are available and optimization of these techniques was the subject of rigorous research, each technique has its own advantages and limitations and the proper isolation method or combination of methods must be chosen on the basis of the research question, quantity and quality and level of purity needed. Table 2 overviews some of the available isolations technique as well as their applications and limitations. One important pre-analytical issue in EV biomarker discovery is the lack of a generally accepted standardization in isolation methods and guidelines related to sample collection and handling for EV studies. Conditions of sample storage, EV isolation methods and subsequent RNA isolation methods can affect downstream RNA and protein profile. For example, Zhou et al. reported that freezing at $-20^{\circ} \mathrm{C}$ lead to a major loss in urinary EVs compared with storing at $-80^{\circ} \mathrm{C}^{90}$. Increasing time between venipuncture and centrifugation has also been reported to induce degradation and rupture of EVs ${ }^{91}$. Another example of a pre-analytical parameter that should be taken into account is the effect of circadian rhythm on EV cargos. For instance, expression of solute carrier family 12 member 3 (also known as $\mathrm{Na}-\mathrm{Cl}$ cotransporter, $\mathrm{NCC}$ ) and prostasin in urinary EVs has been reported to be affected by circadian rhythm ${ }^{92}$.

\section{EVs as therapeutic tools in liver diseases}

The use of EVs, especially the exosome subpopulation, as vehicles for RNA interference (RNAi) and drug delivery has generated considerable attention in the scientific community. 
EVs are bioavailable, biocompatible and resistant to RNases and proteases. ${ }^{93,}, 94$ These characteristics make EVs ideal vehicles for the delivery of drugs, proteins, microRNAs, silencing RNA (siRNA) and other molecules that would otherwise be rapidly degraded (Box 1). EVs provide opportunities to deliver tissue-targeted siRNA and miRNAs to regulate gene expression within target cells. Studies suggest that the liver is a primary site of EV uptake after intravenous delivery ${ }^{45}$, and experimental evidence has shown the potential use of EVs (especially the exosome subfraction) in delivering targeted RNA-based therapies in the context of liver diseases. ${ }^{45,}, 95$ Our group found that EVs can efficiently deliver a miR-155 mimic or inhibitor to macrophages and hepatocytes both in vitro and in vivo. ${ }^{45,95}$ miRNA-155 was previously shown to be an important mediator of liver inflammation in alcoholic hepatitis. ${ }^{96}$ Thus, EVs derived from B cells, which harbour very low levels of endogenous miRNA-155, were used to introduce an exogenous miRNA-155 synthetic mimic and a miRNA-155 inhibitor to RAW macrophages. The transferred miRNA inhibitor and mimic were both active and delivery of the miR-155 inhibitor to RAW macrophages induced functional knock-down of TNF protein production by more than $50 \% .{ }^{95}$ In vivo, EVs successfully delivered an exogenous miR-155 mimic to the liver and isolated hepatocytes in miR-155 knockout mice. ${ }^{45,95}$ Exosome-mediated delivery of RNAi is shown in FIG. 5.

EV-based delivery methods for targeting different pathways involved in liver diseases are faced with several remaining challenges. First, before EVs can be used as therapy in humans, standardization and quality control of EVs will have to adhere to industry and/or regulator standards. This is a challenge together with the cost of large-scale production of EVs. ${ }^{2}$ Second, because EV contents vary based on the type and state of parental cells ${ }^{17}$, the type of EV selection for delivery will be critical and should be selected based on the type of recipient cells and the target molecule. Third, although clinical trials have demonstrated that autologous EVs are well-tolerated after repetitive administration ${ }^{97}$, the clinical therapeutic benefit of EV-based or exosome-based therapy in specific liver diseases awaits randomized controlled clinical trials.

The relevance of EV-mediated signalling in the initiation and progression of liver disease renders EVs a unique class of therapeutics targets. Different possibilities in the clinical setting, although still largely theoretical, include interruption of EV-mediated cross-talk between cells in the diseased liver. Our group showed that EVs have pivotal roles in an alternative route of $\mathrm{HCV}$ cellular transmission and possibly resistance cases of $\mathrm{HCV} .{ }^{46} \mathrm{EV}$ removal from the circulation or inhibition of EV uptake by target recipient cells could be attractive therapeutic options in mitigating HCV transmission via EVs. EVs can be depleted from the circulation using approaches similar to the removal of circulating antibodies by extracorporeal dialysis utilized in autoimmune diseases. ${ }^{98}$ In principal, this approach could be tailored for other liver diseases in which exosomes mediate deleterious paracrine effects. Inhibition of EV uptake is another approach that can be achieved by administration of PPIs. ${ }^{99}$ Administration of a PPI effectively interfered with exosome uptake and prevented transmission of EV-mediated HCV infection in vitro in Huh 7.5 cells. ${ }^{46}$ However, the high concentration of proton pump inhibitors needed in vivo to inhibit EV uptake is a major limitation. 
Studies published in the past few years indicate the involvement of EVs in repairing tissue and organ damage, which can partially explain the paracrine effects reported in the stem cell therapies. ${ }^{100,101}$ The content of EVs can elicit cell recruitment, differentiation and proliferation. ${ }^{101}$ Mesenchymal-stem-cell-derived EVs promote hepatic regeneration in DILI animal models by inducing the IL-6-STAT3 pathway and cell cycle progression. ${ }^{100}$ In a mouse model of liver fibrosis induced by carbon tetrachloride, EVs derived from human umbilical cord mesenchymal stem cells attenuated liver fibrosis, as indicated by reduced surface fibrous capsules, reduced production of collagen type I and type III, reduced serum levels of transforming growth factor $\beta$ and improved serum AST level. ${ }^{102}$ The use of stemcell-derived EVs instead of stem cells engraftment might open new perspectives for hepatic regenerative therapies. This approach would be beneficial from different perspectives. First, using EVs derived from stem cells instead of the cells themselves would remove concerns about aberrant stem cell differentiation, high immune reaction risks and risk of carcinogenesis. ${ }^{103,104}$ Second, immunosuppressive effects associated with certain types of EVs could support liver tissue regeneration. ${ }^{105}$ Third, EVs and exosomes could be isolated from the blood and could be stored while maintaining their biological properties for years ${ }^{104}$, facilitating their use for liver regeneration at later time-points. It is tempting to speculate that exosomes isolated from patients with an early phse of liver disease could be explored for personalized medicine approaches if the patient reaches advanced liver disease. Although EVs offer hope for circumventing the complications associated with the use of viable cells in regenerative medicine, further meticulous investigations are warranted to study the procarcinogenic and anticarcinogenic properties reported for mesenchymal-stemcell-derived EVs. ${ }^{28}$

\section{Conclusion}

EVs contain various biological macromolecules, including proteins, genetic material and lipids, and have pivotal roles in conveying information between different cell types in the liver. EVs can alter function and activate different pathways in recipient cells, which then contribute to the initiation, progression and pathogenesis of different liver diseases.

Circulating EVs are emerging as potential biomarkers of liver disease and in monitoring responses to treatment. EVs could be used for liver disease modification or liver regeneration, and exploiting the inherent ability of EVs to deliver nucleic acids and other drug cargoes to cells in the liver might see EVs emerge as a novel therapeutic approach. Box 1 demonstrates potential projections of the field and future directions by the authors.

\section{Acknowledgments:}

This study was supported by UO1 translational (AA021907/103) and UO1 clinical AA021893-03 to GS.

\section{Biographies:}

Gyongyi Szabo is the Worcester Foundation for Biomedical Research Endowed Chair, Associate Vice Provost for Interprofessional Education and Vice Chair in the Department of Medicine at the University of Massachusetts Medical School, Worcester, MA, USA. She is a physician scientist with active translational and clinical research studies in liver diseases, 
and is an international authority in liver inflammation and immunity. Her research focuses on cellular and molecular mechanisms of inflammation in alcoholic and non-alcoholic liver diseases with the goal to identify and test novel therapeutic approaches for steatohepatitis that could be brought to the clinic. Her recent studies focus on extracellular vesicles and extracellular RNAs in biomarker discovery, disease progression and therapies in liver diseases. She is member of the Hungarian Academy of Sciences and serves on advisory boards of several academic institutions and pharmaceutical companies. She is Immediate Past President of the American Association of the Study of Liver Diseases, the largest professional society in liver diseases and the inaugural Editor-in-Chief of Hepatology Communications.

Fatemeh Momen-Heravi is currently a periodontics and implant surgery resident at Columbia College of Dental Medicine, New York, NY, USA. She joined the Szabo laboratory at the University of Massachusetts Medical School, MA, USA, in 2013 as a postdoctoral researcher after finishing her postdoc at Harvard Medical School, MA, USA. Her research interests include extracellular vesicles, non-coding RNA and gene editing, and she has studied the role of extracellular vesicles in disease pathogenesis and molecular targeting by using RNA inferences. She has worked on different in vitro and in vivo models of disease, including liver diseases and cancers.

\section{References}

1. Kalra $\mathrm{H}$ et al. Vesiclepedia: a compendium for extracellular vesicles with continuous community annotation. PLoS Biol. 10, e1001450 (2012). [PubMed: 23271954]

2. Momen-Heravi $\mathrm{F}$ et al. Current methods for the isolation of extracellular vesicles. Biol. Chem 394, 1253-1262 (2013). [PubMed: 23770532]

3. Lotvall $\mathrm{J}$ et al. Minimal experimental requirements for definition of extracellular vesicles and their functions: a position statement from the International Society for Extracellular Vesicles. J. Extracell Vesicles 3, 26913 (2014). [PubMed: 25536934]

4. Yanez-Mo M et al. Biological properties of extracellular vesicles and their physiological functions. J. Extracell Vesicles 4, 27066 (2015). [PubMed: 25979354]

5. Stoorvogel W, Kleijmeer MJ, Geuze HJ \& Raposo G The biogenesis and functions of exosomes. Traffic 3, 321-330 (2002). [PubMed: 11967126]

6. Raposo G \& Stoorvogel W Extracellular vesicles: exosomes, microvesicles, and friends. J. Cell Biol. 200, 373-383 (2013). [PubMed: 23420871]

7. Simpson RJ \& Mathivanan S Extracellular Microvesicles: The Need for Internationally Recognised Nomenclature and Stringent Purification Criteria J. Proteomics Bioinform 5 (2012).

8. Cocucci E \& Meldolesi J Ectosomes and exosomes: shedding the confusion between extracellular vesicles. Trends Cell Biol. 25, 364-372 (2015). [PubMed: 25683921]

9. Kowal J et al. Proteomic comparison defines novel markers to characterize heterogeneous populations of extracellular vesicle subtypes. Proc. Natl. Acad. Sci. U. S. A 113, E968-77 (2016). [PubMed: 26858453]

10. Hurley JH \& Odorizzi G Get on the exosome bus with ALIX. Nat. Cell Biol 14, 654-655 (2012). [PubMed: 22743708]

11. Vlassov AV, Magdaleno S, Setterquist R \& Conrad R Exosomes: current knowledge of their composition, biological functions, and diagnostic and therapeutic potentials. Biochim. Biophys. Acta 1820, 940-948 (2012). [PubMed: 22503788]

12. Miller IV \& Grunewald TG Tumour-derived exosomes: Tiny envelopes for big stories. Biol. Cell (2015). 
13. Miranda KC et al. Nucleic acids within urinary exosomes/microvesicles are potential biomarkers for renal disease. Kidney Int 78, 191-199 (2010). [PubMed: 20428099]

14. Saha B, Momen-Heravi F, Kodys K \& Szabo G MicroRNA Cargo of Extracellular Vesicles from Alcohol-exposed Monocytes Signals Naive Monocytes to Differentiate into M2 Macrophages. J. Biol. Chem 291, 149-159 (2016). [PubMed: 26527689]

15. Kato S, Kowashi Y \& Demuth DR Outer membrane-like vesicles secreted by Actinobacillus actinomycetemcomitans are enriched in leukotoxin. Microb. Pathog 32, 1-13 (2002). [PubMed: 11782116]

16. Momen-Heravi F, Bala S, Kodys K \& Szabo G Exosomes derived from alcohol-treated hepatocytes horizontally transfer liver specific miRNA-122 and sensitize monocytes to LPS. Sci. Rep 5, 9991 (2015). [PubMed: 25973575]

17. Jia $\mathrm{S}$ et al. Emerging technologies in extracellular vesicle-based molecular diagnostics. Expert Rev. Mol. Diagn 14, 307-321 (2014). [PubMed: 24575799]

18. Julich H, Willms A, Lukacs-Kornek V \& Kornek M Extracellular vesicle profiling and their use as potential disease specific biomarker. Front. Immunol 5, 413 (2014). [PubMed: 25225495]

19. Properzi F, Logozzi M \& Fais S Exosomes: the future of biomarkers in medicine. Biomark Med 7 , 769-778 (2013). [PubMed: 24044569]

20. Alexander $\mathrm{M}$ et al. Exosome-delivered microRNAs modulate the inflammatory response to endotoxin. Nat. Commun 6, 7321 (2015). [PubMed: 26084661]

21. Hoshino A et al. Tumour exosome integrins determine organotropic metastasis. Nature 527, 329335 (2015). [PubMed: 26524530]

22. Lemoinne $\mathrm{S}$ et al. The emerging roles of microvesicles in liver diseases. Nat. Rev. Gastroenterol. Hepatol 11, 350-361 (2014). [PubMed: 24492276]

23. Royo F et al. Transcriptome of extracellular vesicles released by hepatocytes. PLoS One 8 , e68693 (2013). [PubMed: 23874726]

24. Rodriguez-Suarez E et al. Quantitative proteomic analysis of hepatocyte-secreted extracellular vesicles reveals candidate markers for liver toxicity. J. Proteomics 103, 227-240 (2014). [PubMed: 24747303]

25. Masyuk AI, Masyuk TV \& Larusso NF Exosomes in the pathogenesis, diagnostics and therapeutics of liver diseases. J. Hepatol 59, 621-625 (2013). [PubMed: 23557871]

26. Chen L, Chen R, Kemper S, Charrier A \& Brigstock DR Suppression of fibrogenic signaling in hepatic stellate cells by Twist1-dependent microRNA-214 expression: Role of exosomes in horizontal transfer of Twist1. Am. J. Physiol. Gastrointest. Liver Physiol 309, G491-9 (2015). [PubMed: 26229009]

27. Witek RP et al. Liver cell-derived microparticles activate hedgehog signaling and alter gene expression in hepatic endothelial cells. Gastroenterology 136, 320-330.e2 (2009). [PubMed: 19013163]

28. Fonsato $\mathrm{V}$ et al. Human liver stem cell-derived microvesicles inhibit hepatoma growth in SCID mice by delivering antitumor microRNAs. Stem Cells 30, 1985-1998 (2012). [PubMed: 22736596]

29. Deng ZB et al. Intestinal mucus-derived nanoparticle-mediated activation of Wnt/beta-catenin signaling plays a role in induction of liver natural killer T cell anergy in mice. Hepatology 57, 1250-1261 (2013). [PubMed: 22991247]

30. Qu Z et al. Exosomes derived from HCC cells induce sorafenib resistance in hepatocellular carcinoma both in vivo and in vitro. J. Exp. Clin. Cancer Res 35, 159 (2016). [PubMed: 27716356]

31. Conde-Vancells J et al. Characterization and comprehensive proteome profiling of exosomes secreted by hepatocytes. J. Proteome Res 7, 5157-5166 (2008). [PubMed: 19367702]

32. Masyuk AI et al. Biliary exosomes influence cholangiocyte regulatory mechanisms and proliferation through interaction with primary cilia. Am. J. Physiol. Gastrointest. Liver Physiol 299, G990-9 (2010). [PubMed: 20634433]

33. Wang Y et al. Chicken biliary exosomes enhance CD4(+)T proliferation and inhibit ALV-J replication in liver. Biochem. Cell Biol 92, 145-151 (2014). [PubMed: 24697699]

34. Rautou PE et al. Abnormal plasma microparticles impair vasoconstrictor responses in patients with cirrhosis. Gastroenterology 143, 166-76.e6 (2012). [PubMed: 22465620] 
35. Wang R et al. Exosome Adherence and Internalization by Hepatic Stellate Cells Triggers Sphingosine 1-Phosphate-dependent Migration. J. Biol. Chem 290, 30684-30696 (2015). [PubMed: 26534962]

36. Szabo G, Saha B \& Ambade A in Hepatology 7th Edition Zskim and Boyers (eds. Boyer T, Sanyal A, Terrault N \& Lindor K) (In Press, 2016).

37. Robbins PD \& Morelli AE Regulation of immune responses by extracellular vesicles. Nat. Rev. Immunol 14, 195-208 (2014). [PubMed: 24566916]

38. Chatila TA \& Williams CB Regulatory T cells: exosomes deliver tolerance. Immunity 41, 3-5 (2014). [PubMed: 25035945]

39. O’Neill HC \& Quah BJ Exosomes secreted by bacterially infected macrophages are proinflammatory. Sci. Signal 1, pe 8 (2008).

40. Nojima $\mathrm{H}$ et al. Hepatocyte exosomes mediate liver repair and regeneration via sphingosine-1phosphate. J. Hepatol 64, 60-68 (2016). [PubMed: 26254847]

41. Simons M \& Raposo G Exosomes--vesicular carriers for intercellular communication. Curr. Opin. Cell Biol 21, 575-581 (2009). [PubMed: 19442504]

42. Mulcahy LA, Pink RC \& Carter DR Routes and mechanisms of extracellular vesicle uptake. J. Extracell Vesicles 3, 10.3402/jev.v3.24641. eCollection 2014 (2014).

43. Imai $\mathrm{T}$ et al. Macrophage-dependent clearance of systemically administered B16BL6-derived exosomes from the blood circulation in mice. J. Extracell Vesicles 4, 26238 (2015). [PubMed: 25669322]

44. Bala $\mathrm{S}$ et al. Increased microRNA-155 expression in the serum and peripheral monocytes in chronic HCV infection. J. Transl. Med 10, 151-5876-10-151 (2012).

45. Bala $\mathrm{S}$ et al. Biodistribution and function of extracellular miRNA-155 in mice. Sci. Rep 5, 10721 (2015). [PubMed: 26024046]

46. Bukong TN, Momen-Heravi F, Kodys K, Bala S \& Szabo G Exosomes from Hepatitis C Infected Patients Transmit HCV Infection and Contain Replication Competent Viral RNA in Complex with Ago2-miR122-HSP90. PLoS Pathog 10, e1004424 (2014). [PubMed: 25275643]

47. Dreux $M$ et al. Short-range exosomal transfer of viral RNA from infected cells to plasmacytoid dendritic cells triggers innate immunity. Cell. Host Microbe 12, 558-570 (2012). [PubMed: 23084922]

48. Bhattarai $\mathrm{N}$ et al. GB virus $\mathrm{C}$ particles inhibit $\mathrm{T}$ cell activation via envelope $\mathrm{E} 2$ protein-mediated inhibition of TCR signaling. J. Immunol 190, 6351-6359 (2013). [PubMed: 23686495]

49. Chivero ET \& Stapleton JT Tropism of human pegivirus (formerly known as GB virus C/hepatitis $\mathrm{G}$ virus) and host immunomodulation: insights into a highly successful viral infection. J. Gen. Virol 96, 1521-1532 (2015). [PubMed: 25667328]

50. Fusegawa $\mathrm{H}$ et al. Platelet activation in patients with chronic hepatitis C. Tokai J. Exp. Clin. Med 27, 101-106 (2002). [PubMed: 12713014]

51. Li J et al. Exosomes mediate the cell-to-cell transmission of IFN-alpha-induced antiviral activity. Nat. Immunol 14, 793-803 (2013). [PubMed: 23832071]

52. Yang J, Liu Z \& Xiao TS Post-translational regulation of inflammasomes. Cell. Mol. Immunol 14(1), 65-79 (2017). [PubMed: 27345727]

53. Saha B, Kodys K \& Szabo G Hepatitis C Virus-Induced Monocyte Differentiation Into Polarized M2 Macrophages Promotes Stellate Cell Activation via TGF- $\beta$. Cellular and Molecular Gastroenterology and Hepatology 2(3), 302-316 (2016). [PubMed: 28090562]

54. Povero D et al. Circulating extracellular vesicles with specific proteome and liver microRNAs are potential biomarkers for liver injury in experimental fatty liver disease. PLoS One 9, e113651 (2014). [PubMed: 25470250]

55. Kakazu E, Mauer AS, Yin M \& Malhi H Hepatocytes release ceramide-enriched pro-inflammatory extracellular vesicles in an IRE1alpha-dependent manner. J. Lipid Res 57, 233-245 (2016). [PubMed: 26621917]

56. Heinrich LF, Andersen DK, Cleasby ME \& Lawson C Long-term high fat feeding of rats results in increased numbers of circulating microvesicles with pro-inflammatory effects on endothelial cells. Br. J. Nutr 113, 1704-1711 (2015). [PubMed: 25880162] 
57. Povero D et al. Lipid-induced toxicity stimulates hepatocytes to release angiogenic microparticles that require Vanin-1 for uptake by endothelial cells. Sci. Signal 6, ra88 (2013).

58. Kornek $\mathrm{M}$ et al. Circulating microparticles as disease-specific biomarkers of severity of inflammation in patients with hepatitis C or nonalcoholic steatohepatitis. Gastroenterology 143, 448-458 (2012). [PubMed: 22537612]

59. Hirsova P et al. Lipid-Induced Signaling Causes Release of Inflammatory Extracellular Vesicles From Hepatocytes. Gastroenterology 150, 956-967 (2016). [PubMed: 26764184]

60. Ibrahim SH et al. Mixed lineage kinase 3 mediates release of C-X-C motif ligand 10-bearing chemotactic extracellular vesicles from lipotoxic hepatocytes. Hepatology 63, 731-744 (2016). [PubMed: 26406121]

61. Verma VK et al. Alcohol stimulates macrophage activation through caspase-dependent hepatocyte derived release of CD40L containing extracellular vesicles. J. Hepatol 64, 651-660 (2016). [PubMed: 26632633]

62. Momen-Heravi $\mathrm{F}$ et al. Increased number of circulating exosomes and their microRNA cargos are potential novel biomarkers in alcoholic hepatitis. J. Transl. Med 13, 261-015-0623-9 (2015).

63. Holman NS, Mosedale M, Wolf KK, LeCluyse EL \& Watkins PB Subtoxic Alterations in Hepatocyte-Derived Exosomes: An Early Step in Drug-Induced Liver Injury? Toxicol. Sci 151, 365-375 (2016). [PubMed: 26962055]

64. Bala $\mathrm{S}$ et al. Circulating microRNAs in exosomes indicate hepatocyte injury and inflammation in alcoholic, drug-induced, and inflammatory liver diseases. Hepatology 56, 1946-1957 (2012). [PubMed: 22684891]

65. Ward J et al. Circulating microRNA profiles in human patients with acetaminophen hepatotoxicity or ischemic hepatitis. Proc. Natl. Acad. Sci. U. S. A 111, 12169-12174 (2014). [PubMed: 25092309]

66. Sugimachi $\mathrm{K}$ et al. Identification of a bona fide microRNA biomarker in serum exosomes that predicts hepatocellular carcinoma recurrence after liver transplantation. Br. J. Cancer 112, 532538 (2015). [PubMed: 25584485]

67. Kogure T, Lin WL, Yan IK, Braconi C \& Patel T Intercellular nanovesicle-mediated microRNA transfer: a mechanism of environmental modulation of hepatocellular cancer cell growth. Hepatology 54, 1237-1248 (2011). [PubMed: 21721029]

68. Lv LH et al. Anticancer drugs cause release of exosomes with heat shock proteins from human hepatocellular carcinoma cells that elicit effective natural killer cell antitumor responses in vitro. J. Biol. Chem 287, 15874-15885 (2012). [PubMed: 22396543]

69. Bruno $\mathrm{S}$ et al. Microvesicles derived from human bone marrow mesenchymal stem cells inhibit tumor growth. Stem Cells Dev 22, 758-771 (2013). [PubMed: 23034046]

70. Chiba M, Kimura M \& Asari S Exosomes secreted from human colorectal cancer cell lines contain mRNAs, microRNAs and natural antisense RNAs, that can transfer into the human hepatoma HepG2 and lung cancer A549 cell lines. Oncol. Rep 28, 1551-1558 (2012). [PubMed: 22895844]

71. Costa-Silva B et al. Pancreatic cancer exosomes initiate pre-metastatic niche formation in the liver. Nat. Cell Biol 17, 816-826 (2015). [PubMed: 25985394]

72. Wang $X$ et al. Investigation of the roles of exosomes in colorectal cancer liver metastasis. Oncol. Rep 33, 2445-2453 (2015). [PubMed: 25760247]

73. Aithal GP, Guha N, Fallowfield J, Castera L \& Jackson AP Biomarkers in liver disease: emerging methods and potential applications. Int. J. Hepatol 2012, 437508 (2012). [PubMed: 23209913]

74. Patel K, Bedossa P \& Castera L Diagnosis of liver fibrosis: present and future. Semin. Liver Dis 35, 166-183 (2015). [PubMed: 25974902]

75. Kim WR, Flamm SL, Di Bisceglie AM, Bodenheimer HC \& Public Policy Committee of the American Association for the Study of Liver Disease. Serum activity of alanine aminotransferase (ALT) as an indicator of health and disease. Hepatology 47, 1363-1370 (2008). [PubMed: 18366115]

76. Tang MK \& Wong AS Exosomes: Emerging biomarkers and targets for ovarian cancer. Cancer Lett 367, 26-33 (2015). [PubMed: 26189430]

77. Mahmoudi K, Ezrin A \& Hadjipanayis C Small extracellular vesicles as tumor biomarkers for glioblastoma. Mol. Aspects Med 45, 97-102 (2015). [PubMed: 26118341] 
78. De Toro J, Herschlik L, Waldner C \& Mongini C Emerging roles of exosomes in normal and pathological conditions: new insights for diagnosis and therapeutic applications. Front. Immunol 6, 203 (2015). [PubMed: 25999947]

79. Lambertz U et al. Small RNAs derived from tRNAs and rRNAs are highly enriched in exosomes from both old and new world Leishmania providing evidence for conserved exosomal RNA Packaging. BMC Genomics 16, 151-015-1260-7 (2015).

80. Pant S, Hilton H \& Burczynski ME The multifaceted exosome: biogenesis, role in normal and aberrant cellular function, and frontiers for pharmacological and biomarker opportunities. Biochem. Pharmacol 83, 1484-1494 (2012). [PubMed: 22230477]

81. Looze $\mathrm{C}$ et al. Proteomic profiling of human plasma exosomes identifies PPARgamma as an exosome-associated protein. Biochem. Biophys. Res. Commun 378, 433-438 (2009). [PubMed: 19028452]

82. Moratti E, Vezzalini M, Tomasello L, Giavarina D \& Sorio C Identification of protein tyrosine phosphatase receptor gamma extracellular domain (sPTPRG) as a natural soluble protein in plasma. PLoS One 10, e0119110 (2015). [PubMed: 25775014]

83. Charrier A et al. Exosomes mediate intercellular transfer of pro-fibrogenic connective tissue growth factor (CCN2) between hepatic stellate cells, the principal fibrotic cells in the liver. Surgery 156, 548-555 (2014). [PubMed: 24882759]

84. Welker MW et al. Soluble serum CD81 is elevated in patients with chronic hepatitis C and correlates with alanine aminotransferase serum activity. PLoS One 7, e30796 (2012). [PubMed: 22355327]

85. Butler SL et al. The antigen for Hep Par 1 antibody is the urea cycle enzyme carbamoyl phosphate synthetase 1. Lab. Invest 88, 78-88 (2008). [PubMed: 18026163]

86. Brodsky SV et al. Dynamics of circulating microparticles in liver transplant patients. J. Gastrointestin Liver Dis 17, 261-268 (2008). [PubMed: 18836617]

87. Conde-Vancells J et al. Candidate biomarkers in exosome-like vesicles purified from rat and mouse urine samples. Proteomics Clin. Appl 4, 416-425 (2010). [PubMed: 20535238]

88. Wang $\mathrm{H}$ et al. Expression of serum exosomal microRNA-21 in human hepatocellular carcinoma. Biomed. Res. Int 2014, 864894 (2014). [PubMed: 24963487]

89. Szabo G \& Bala S MicroRNAs in liver disease. Nat. Rev. Gastroenterol. Hepatol 10, 542-552 (2013). [PubMed: 23689081]

90. Zhou $\mathrm{H}$ et al. Collection, storage, preservation, and normalization of human urinary exosomes for biomarker discovery. Kidney Int 69, 1471-1476 (2006). [PubMed: 16501490]

91. Ayers L et al. Measurement of circulating cell-derived microparticles by flow cytometry: sources of variability within the assay. Thromb. Res 127, 370-377 (2011). [PubMed: 21257195]

92. Castagna A et al. Circadian exosomal expression of renal thiazide-sensitive $\mathrm{NaCl}$ cotransporter (NCC) and prostasin in healthy individuals. Proteomics Clin. Appl 9, 623-629 (2015). [PubMed: 25931204]

93. Johnsen $\mathrm{KB}$ et al. A comprehensive overview of exosomes as drug delivery vehicles - endogenous nanocarriers for targeted cancer therapy. Biochim. Biophys. Acta 1846, $75-87$ (2014). [PubMed: 24747178]

94. Marcus ME \& Leonard JN FedExosomes: Engineering Therapeutic Biological Nanoparticles that Truly Deliver. Pharmaceuticals (Basel) 6, 659-680 (2013). [PubMed: 23894228]

95. Momen-Heravi F, Bala S, Bukong T \& Szabo G Exosome-mediated delivery of functionally active miRNA-155 inhibitor to macrophages. Nanomedicine 10(7), 1517-27 (2014). [PubMed: 24685946]

96. Bala S et al. Up-regulation of microRNA-155 in macrophages contributes to increased tumor necrosis factor TNFa production via increased mRNA half-life in alcoholic liver disease. J. Biol. Chem 286, 1436-1444 (2011). [PubMed: 21062749]

97. Escudier B et al. Vaccination of metastatic melanoma patients with autologous dendritic cell (DC) derived-exosomes: results of thefirst phase I clinical trial. J. Transl. Med 3, 10 (2005). [PubMed: 15740633]

98. Marleau AM, Chen CS, Joyce JA \& Tullis RH Exosome removal as a therapeutic adjuvant in cancer. J. Transl. Med 10, 134-5876-10-134 (2012). 
99. Parolini I et al. Microenvironmental pH is a key factor for exosome traffic in tumor cells. J. Biol. Chem 284, 34211-34222 (2009). [PubMed: 19801663]

100. Tan CY et al. Mesenchymal stem cell-derived exosomes promote hepatic regeneration in druginduced liver injury models. Stem Cell. Res. Ther 5, 76 (2014). [PubMed: 24915963]

101. De Jong OG, Van Balkom BW, Schiffelers RM, Bouten CV \& Verhaar MC Extracellular vesicles: potential roles in regenerative medicine. Front. Immunol 5, 608 (2014). [PubMed: 25520717]

102. $\mathrm{Li} \mathrm{T}$ et al. Exosomes derived from human umbilical cord mesenchymal stem cells alleviate liver fibrosis. Stem Cells Dev 22, 845-854 (2013). [PubMed: 23002959]

103. Navarro-Alvarez N, Soto-Gutierrez A \& Kobayashi N Stem cell research and therapy for liver disease. Curr. Stem Cell. Res. Ther 4, 141-146 (2009). [PubMed: 19442198]

104. Fleury A, Martinez MC \& Le Lay S Extracellular vesicles as therapeutic tools in cardiovascular diseases. Front. Immunol 5, 370 (2014). [PubMed: 25136343]

105. Chaput N \& Thery C Exosomes: immune properties and potential clinical implementations. Semin. Immunopathol 33, 419-440 (2011). [PubMed: 21174094]

106. Biomarkers Definitions Working Group. Biomarkers and surrogate endpoints: preferred definitions and conceptual framework. Clin. Pharmacol. Ther 69, 89-95 (2001). [PubMed: 11240971]

107. Strimbu K \& Tavel JA What are biomarkers? Curr. Opin. HIV. AIDS 5, 463-466 (2010). [PubMed: 20978388]

108. Yuana Y, Levels J, Grootemaat A, Sturk A \& Nieuwland R Co-isolation of extracellular vesicles and high-density lipoproteins using density gradient ultracentrifugation. J. Extracell Vesicles 3, 10.3402/jev.v3.23262. eCollection 2014 (2014).

109. Witwer KW et al. Standardization of sample collection, isolation and analysis methods in extracellular vesicle research. J. Extracell Vesicles 2, 10.3402/jev.v2i0.20360. eCollection 2013 (2013).

110. Tauro BJ et al. Comparison of ultracentrifugation, density gradient separation, and immunoaffinity capture methods for isolating human colon cancer cell line LIM1863-derived exosomes. Methods 56, 293-304 (2012). [PubMed: 22285593]

111. Thery C, Amigorena S, Raposo G \& Clayton A Isolation and characterization of exosomes from cell culture supernatants and biological fluids. Curr. Protoc. Cell. Biol. Chapter 3, Unit 3.22 (2006).

112. Liga A, Vliegenthart AD, Oosthuyzen W, Dear JW \& Kersaudy-Kerhoas M Exosome isolation: a microfluidic road-map. Lab. Chip 15, 2388-2394 (2015). [PubMed: 25940789]

113. Wang $\mathrm{Z}$ et al. Ciliated micropillars for the microfluidic-based isolation of nanoscale lipid vesicles. Lab. Chip 13, 2879-2882 (2013). [PubMed: 23743667] 


\section{Box 1}

\section{Future research directions}

Common definitions of EVs types, specific isolation techniques and characterization of EVs, exosomes and microvesicles have led to greatly expanding research into EVs in the past few years. Application of '-omics' technologies such as proteomics, lipidomics and transcriptomics have advanced EV studies to promise new understanding of liver and biliary diseases.

\section{Research areas for the role of EVs in liver disease}

- $\quad$ exploring the biogenesis and biodistribution of EVs

- understanding the role and potential of EVs in intercellular and inter-organ communication

- the use of EVs as biomarkers and non-invasive diagnostic tools

- $\quad$ exploiting EVs as therapeutic tools and delivery systems.

The comprehensive understanding of EVs and their subtypes and cargoes in liver diseases should lead to the development of EV-based biomarkers (liquid biopsy) to aid disease diagnosis. EVs should be exploited in assessment of disease prognosis, stratification of patients and monitoring of disease progression and/or treatment response in the context of personalized medicine. Future studies for EV-based biomarker discovery should use multi-analytical approaches in independent large cohorts, with comparison against current diagnostic methods and biomarkers to identify molecular signatures associated with different liver diseases. Such discoveries should pinpoint potential targets of pharmacological intervention in liver disease. Given the complexity of cells that play roles in the initiation and progression of liver disease, a comprehensive reference profile for cell-specific and tissue-specific EVs and their molecular signatures should be produced. On the basis of studies indicating that exosomes released from one cell type in the liver can modulate the function and/or reprogram other cell types within the liver, additional exploration of EVs in intercellular communication and disease progression is warranted. Finally, the stability and easy cellular uptake of exosomes makes them attractive for their potential as delivery vehicles in therapeutic interventions. Many challenges remain, including the inability to track $\mathrm{EV}$ production and distribution in vivo, the need for better markers to assess specificity in, the cell-of-origin for EVs and the requirement for standardization in diagnostic and therapeutic applications of EVs. 


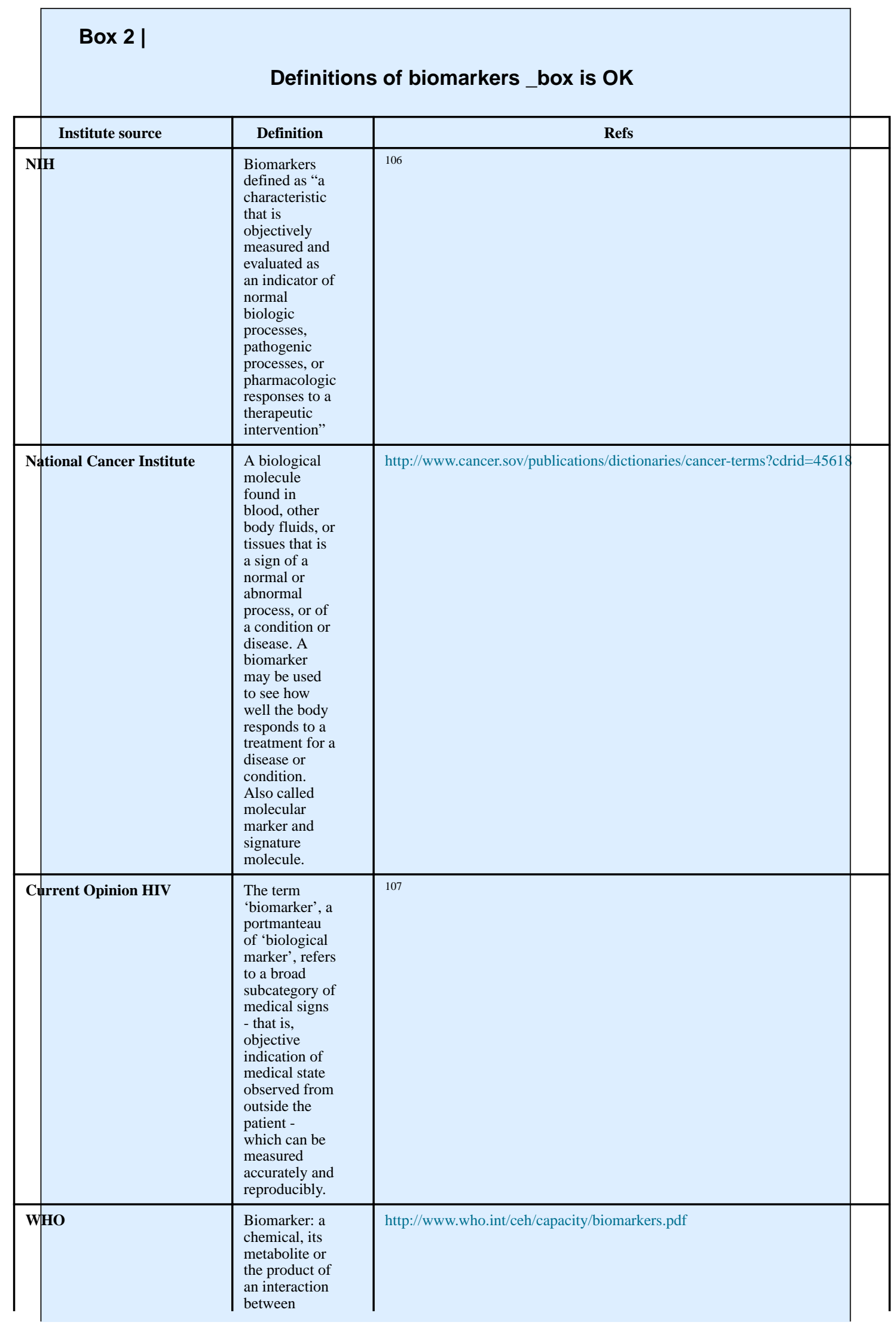

Nat Rev Gastroenterol Hepatol. Author manuscript; available in PMC 2019 February 19. 


\begin{tabular}{|c|c|c|}
\hline Institute source & Definition & Refs \\
\hline & $\begin{array}{l}\text { chemical and } \\
\text { some target } \\
\text { molecule or } \\
\text { cell that is } \\
\text { measured in } \\
\text { the human } \\
\text { body. }\end{array}$ & \\
\hline
\end{tabular}




\section{Key Points:}

- Extracellular vesicles (EVs) are secreted by various liver cell types to the extracellular space and circulation

- $\quad$ Coated with a lipid bilayer, EV cargo contains proteins, lipids and nucleic acids

- $\quad$ The EV cargo represents a snapshot of the parental cell at the time of release; cargo can change depending on the stimulation status and/or differentiation stage of the cell

- $\quad$ EVs are explored as biomarkers of disease and might also represent therapeutic targets and vehicles for therapeutic delivery

- EVs can interact with different cells in the liver through specific receptors and cellular uptake

- Increased levels of circulating EVs have been found in alcoholic liver disease, $\mathrm{NASH}$, viral hepatitis, drug-induced liver injury and hepatocellular carcinoma 


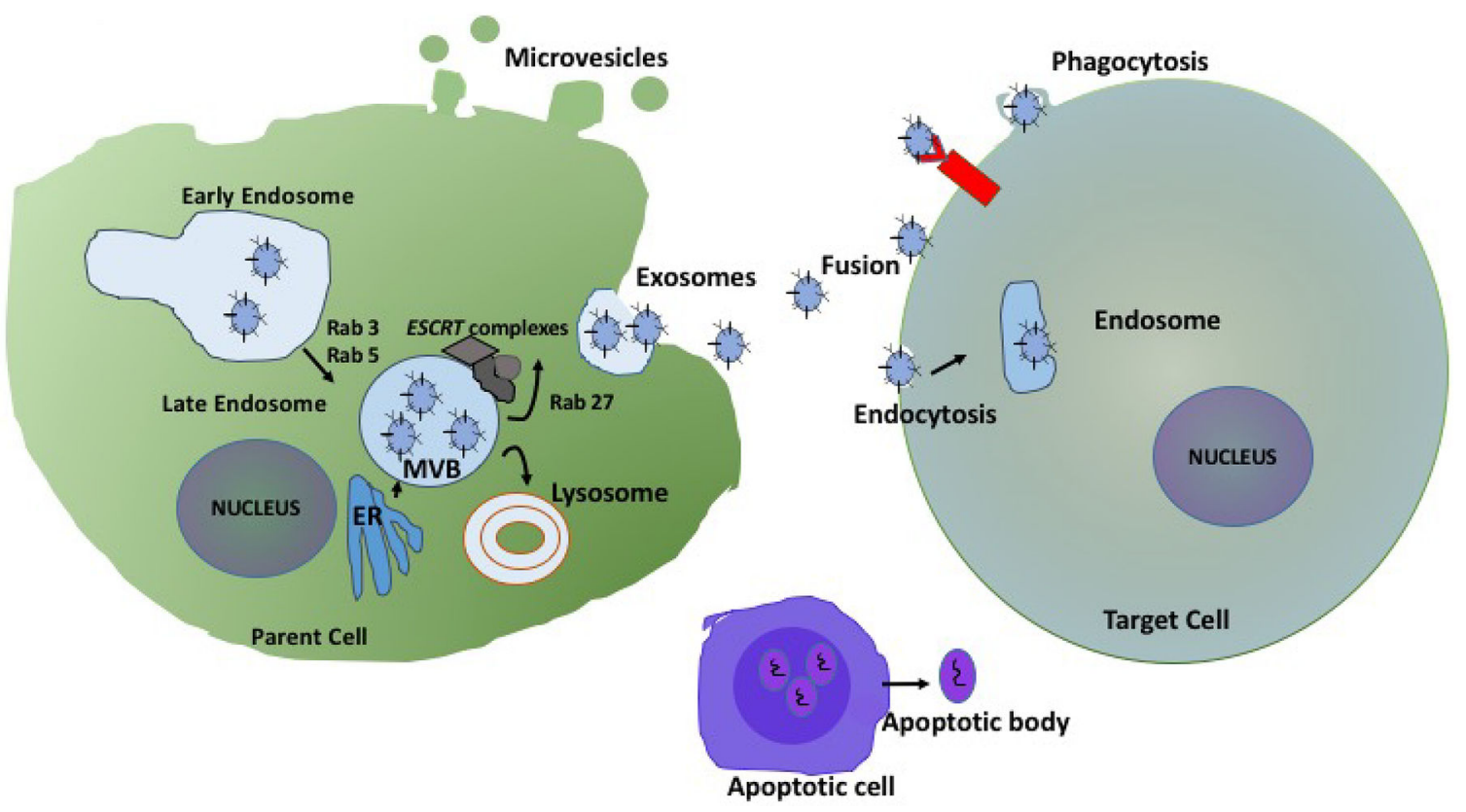

Figure 1 |. Extracellular vesicles biogenesis and release.

Early endosomes are formed as a result of endocytosis at the plasma membrane (1). Rab5 and Rab3 mediate formation of early endosomes. Early endosomes maturate to multivesicular bodies (MVB) (2), which lead to formation of exosomes. The components of the endosomal sorting complex required for transport (ESCRT) complexes are involved in MVB and exosome biogenesis. Rab27 proteins facilitate MVB trafficking and docking on the plasma membrane, leading to MVB exocytosis and exosomes release (3). Microvesicles forms as a result of blebbing of plasma membrane. Extensive plasma membrane blebbing occurs at the plasma membrane of apoptotic cells and leads to the formation of apoptotic bodies. ER, endoplasmic reticulum; ESCRT, endosomal sorting complexes required for transport; MVB, Multivesicular bodies. 


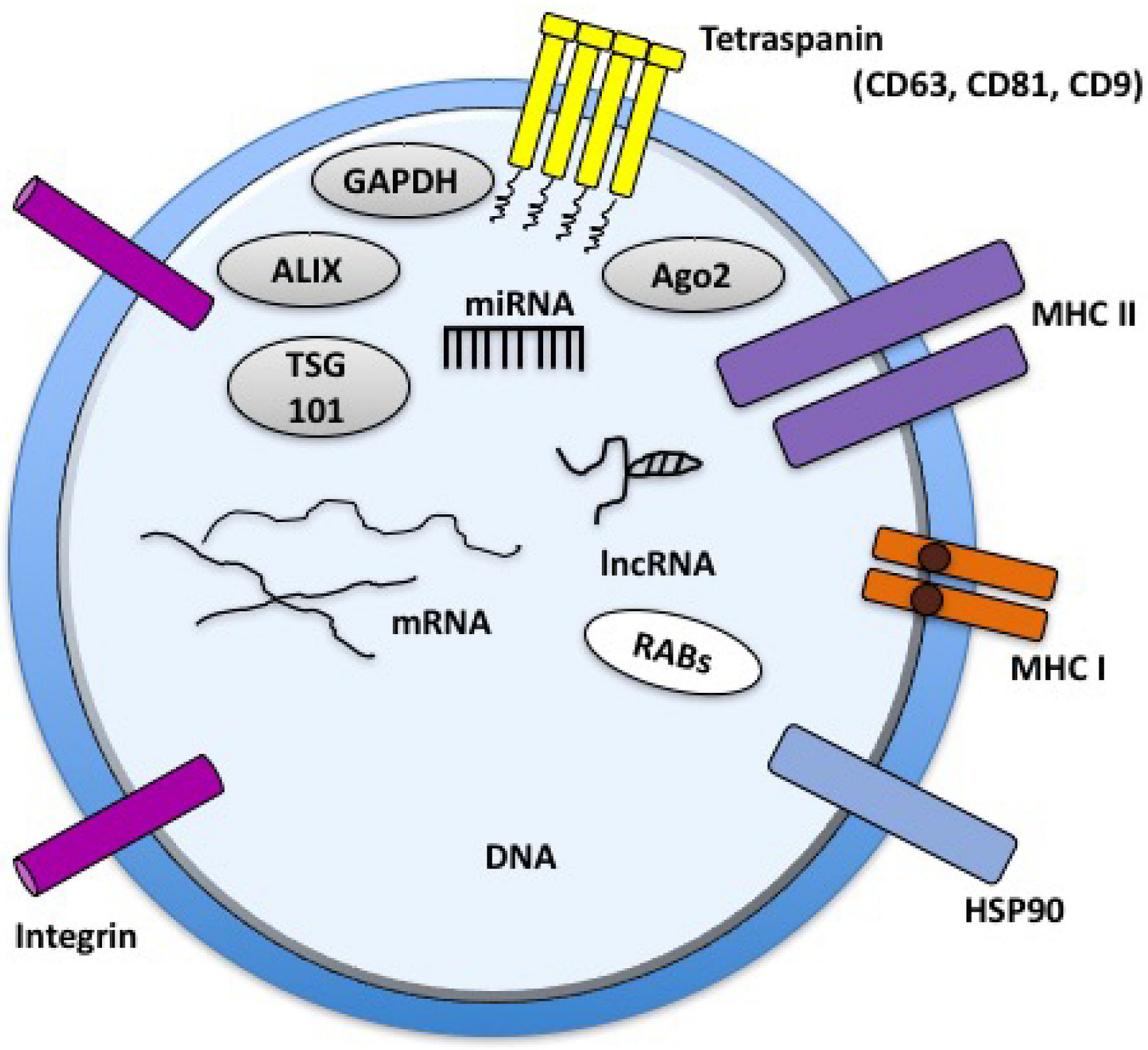

Figure 2 |. Exosome composition.

Schematic representation of exosomes and example of their molecular cargos. Ago2, Argonaute 2; ALIX, ALG-2-interacting protein $\times$ (also known as programmed cell death 6interacting protein); GAPDH, glyceraldehyde-3-phosphate dehydrogenase; HSP90, heat shock protein 90; lncRNA, long non-coding RNA; MHC1, major histocompatibility complex class 1; MHC2, major histocompatibility complex class 2; RABs, Ras-related proteins; TSG101, tumor susceptibility gene 101. 

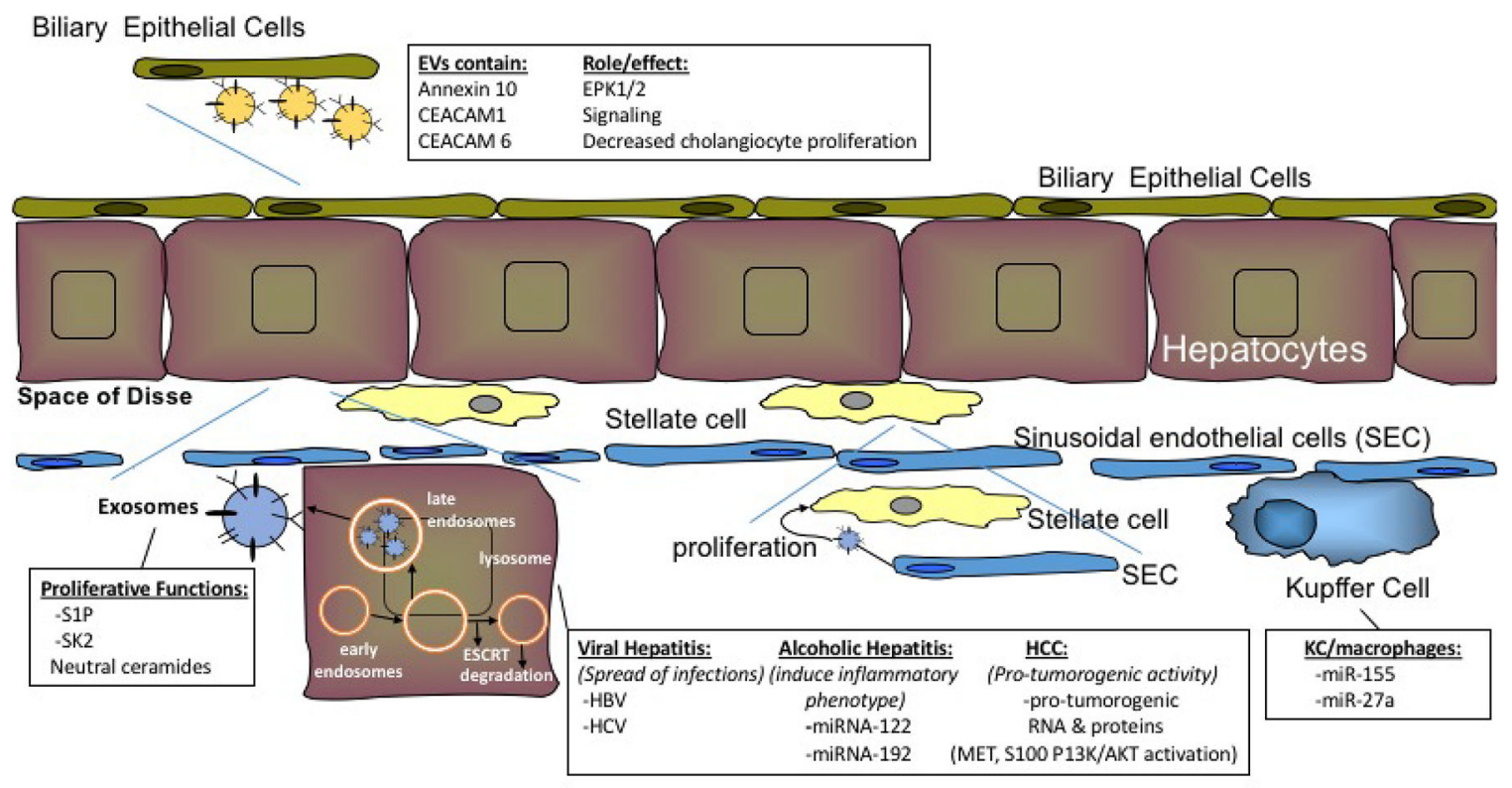

Figure 3 |. Extracellular vesicle (EV) biogenesis and functional role of EVs in liver physiology and pathology.

Biliary EVs can exert functional effects in various signaling pathways including protein kinase activation and decreased cholangiocyte proliferation. Exosomes secreted by hepatocytes induce proliferative function in liver regeneration and play a role in spread of infections in viral hepatitis. In alcoholic hepatitis, hepatocyte derived exosomes contain different miRNAs which induces hyperinflammatory phenotype. In hepatocellular carcinoma exosomes showed pro-tumorigenic activity associated with tumor progression CEACAM1, Carcinoembryonic antigen-related cell adhesion molecule 1; CEACAM6, Carcinoembryonic antigen-related cell adhesion molecule 6; EPK, Eukaryotic protein kinase; ESCRT, Endosomal sorting complexes required for transport; miR, microRNA; S1P, Sphingosine-1-phosphate. SEC, sinusoidal endothelial cells; SK2, sphingosine kinase 2 


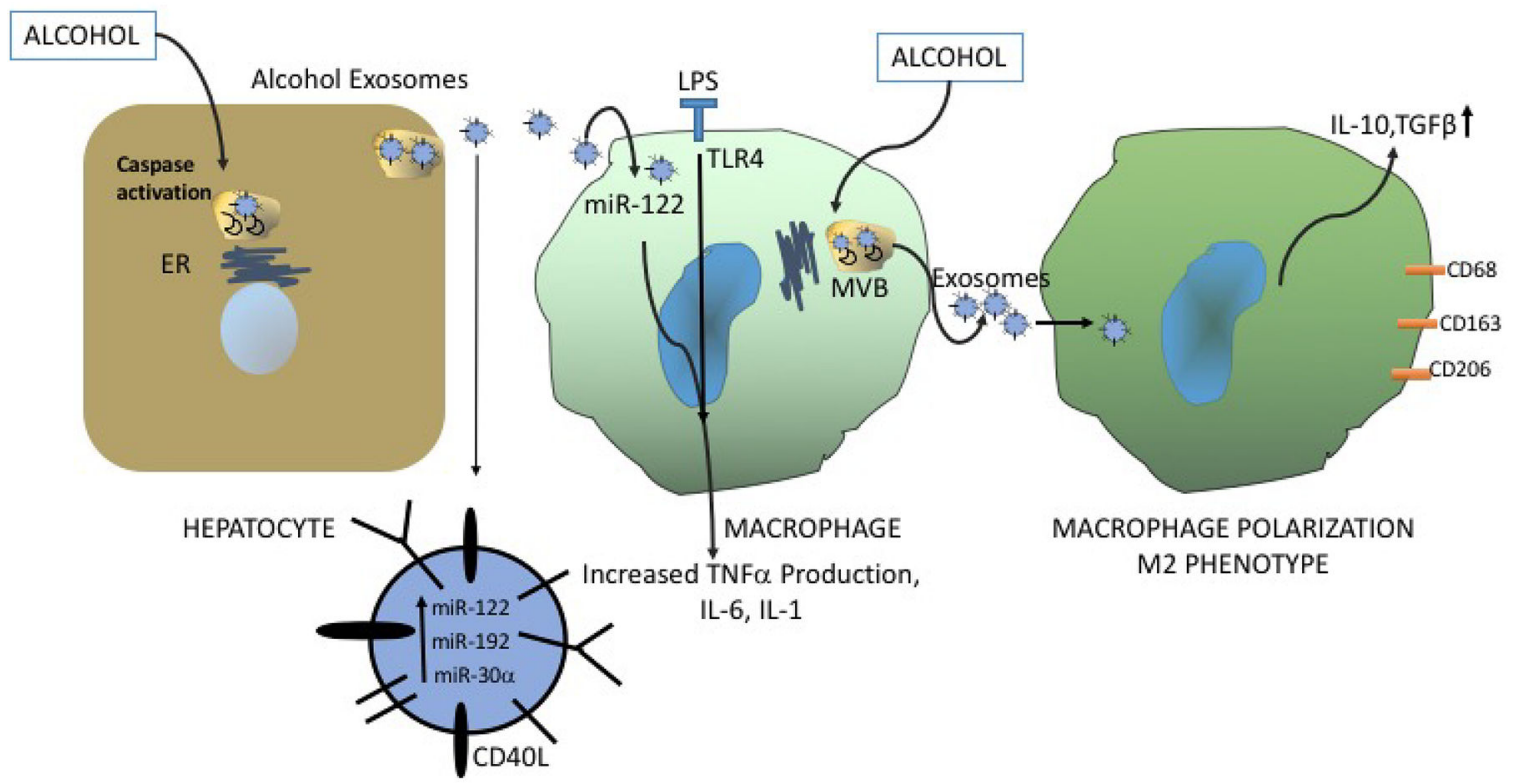

Figure $4 \mid$. Role of exosomes in the pathogenesis of alcoholic hepatitis.

Ethanol induces increased hepatocyte secretion of extracellular vesicles (EVs) harboring elevated levels of microRNA (miR)-122, miR-192 and miR-30a. Alcohol can activate cell regulatory networks controlling inflammation and cell death including caspases which can lead to activation of apoptosis pathways and increase in exosome production. miR-122 sensitizes macrophages to lipopolysaccharide (LPS) stimulation and induces an augmented pro-inflammatory profile. Alcohol increases secretion of EVs, which are taken up by naive monocytes and induce an M2 macrophage phenotype, as indicated by M2 surface markers (CD68, CD163 and CD206) and increased levels of IL-10 and transforming growth factor $\beta$ (TGF $\beta$ ). EVs derived from hepatocytes contain CD40L, and after being taken up by monocytes these EVs promote macrophage activation, contributing to inflammation in alcoholic hepatitis. ER, endoplasmic reticulum; MVB, multivesicular body; TLR4, Toll-like receptor 4 . 


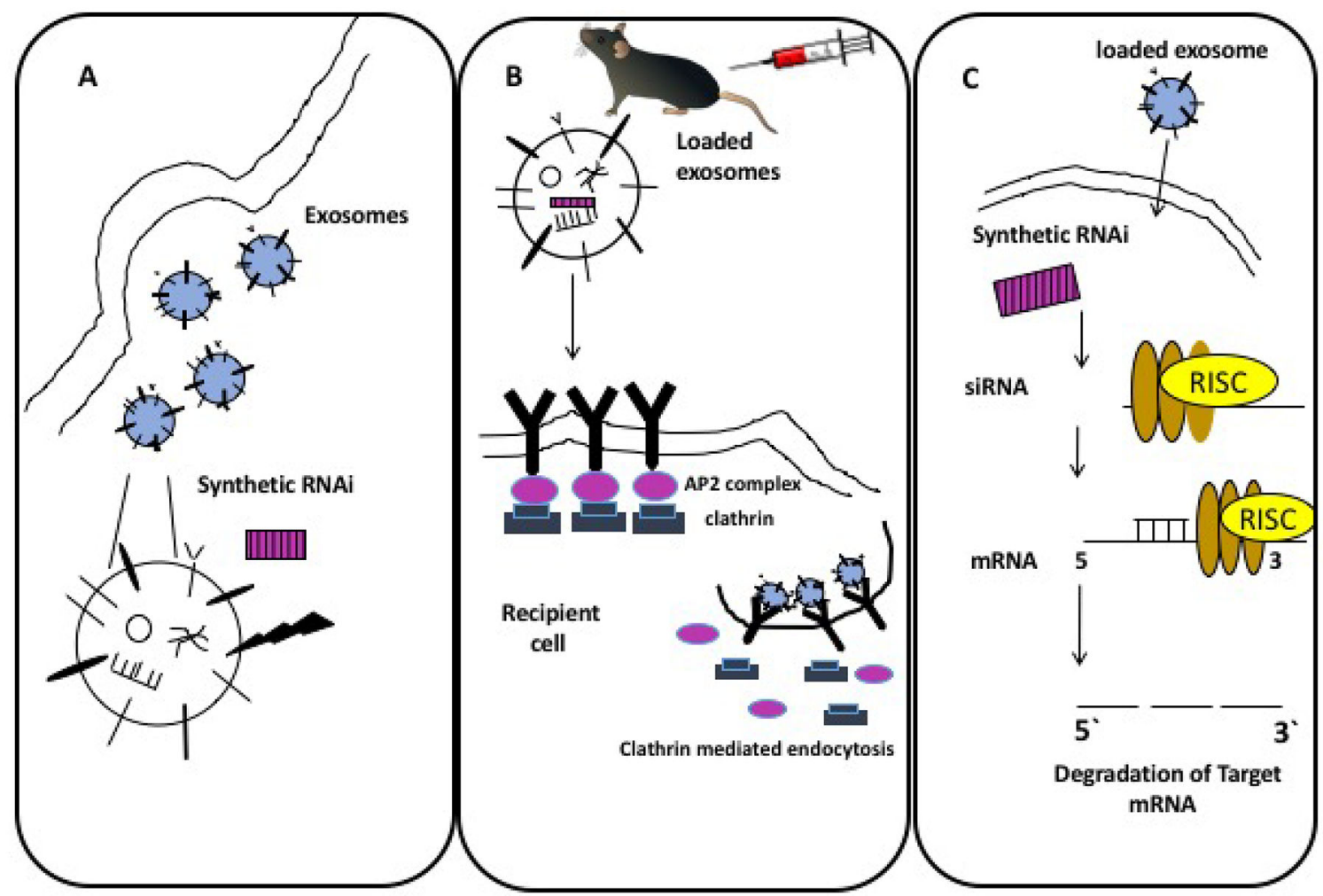

Figure 5 |. Exosomes for delivery of RNA interference (RNAi) therapeutics.

a Synthetic RNAi can be introduced to the exosomes by electroporation. The protocol for loading of exosomes should be optimized for the special target, cargo and model. After loading, exosomes should be re-isolated and the loading efficacy of RNAi should be established and be optimized if necessary $\mathbf{b}$ |Exosomes are injected and can be taken up by different liver cells by clathrin-mediated endocytosis. Introduction of targeting moieties (such as introduction of the APOE to the exosomal lipid bilayer for more rapid and targeted delivery to the hepatocytes) can facilitate the uptake of exosomes by specific liver cells. EVs/Exosomes can deliver their cargo after cellular uptake. $\mathbf{c} \mid \mathrm{EVs} /$ Exosomes deliver synthetic RNAi cargo to the cells. Transferred RNAi can use the host cell RISC complex or exosome- delivered RISC complex. RISC complex to induce degradation of cleavage of target mRNA. AP2, adaptor protein complex 2; RISC, RNA-induced silencing complex 
Table 1.

EV cargo as suggested biomarkers of different liver diseases HCC, hepatocellular carcinoma; Hep par 1, hepatocyte paraffin 1; miRNA, microRNA;

\begin{tabular}{|c|c|c|c|c|c|}
\hline Type of liver disease & Species & $\begin{array}{l}\text { Type of biological } \\
\text { fluid }\end{array}$ & Content of EVs & Control group & Refs \\
\hline \multicolumn{6}{|c|}{ Nucleic acid-based biomarkers } \\
\hline Alcoholic hepatitis & Mice & Serum, Plasma & $\begin{array}{l}\uparrow m i R-122 \\
\uparrow m i R N A-155\end{array}$ & Pair-fed mice & 64 \\
\hline Alcoholic hepatitis & Human & Serum & $\uparrow \mathrm{miR}-122$ & $\begin{array}{l}\text { Healthy individuals, patients } \\
\text { own baseline before binge }\end{array}$ & 16 \\
\hline HCC & Human & Serum & $\downarrow$ miR-718 & Healthy individuals & 66 \\
\hline HCC & Human & Serum & $\uparrow \mathrm{miR}-21$ & $\begin{array}{l}\text { Healthy individuals, patients } \\
\text { with HB V }\end{array}$ & 88 \\
\hline Liver fibrosis & Mice & Serum & $\begin{array}{l}\text { Twistl, miR-214, CCN2 } \\
\text { increased }\end{array}$ & Control mice & 26 \\
\hline Alcoholic hepatitis & Mice & Serum & $\begin{array}{l}\text { miR-122, miRNA-192, } \\
\text { miRNA-30a, increased }\end{array}$ & Control mice & 62 \\
\hline Hepatitis C & Human & Serum & $\begin{array}{l}\text { miR-122, miRNA-134, } \\
\text { miRNA-424-3p, miRNA } \\
629-5 p \text {, increased }\end{array}$ & Healthy individuals & 46 \\
\hline \multicolumn{6}{|l|}{ Protein-based biomarkers } \\
\hline Liver injury & Human & Serum, Plasma & $\uparrow$ sPTPRG & Subjects without liver injury & 82 \\
\hline Hepatitis C & Human & Serum & $\uparrow$ soluble CD81 & Healthy individuals & 84 \\
\hline $\begin{array}{l}\text { HCC and hepatitis C } \\
\text { cirrhosis }\end{array}$ & Human & Plasma & $\uparrow$ Hep par 1 & $\begin{array}{c}\text { Hepatitis C cirrhosis without } \\
\text { HCC }\end{array}$ & 86 \\
\hline $\begin{array}{l}\text { Acute liver injury (D- } \\
\text { galactosamine) }\end{array}$ & Rats & Urine & $\begin{array}{l}\text { CD26, CD81, Slc3A1 and } \\
\text { CD10 }\end{array}$ & Control rats & 87 \\
\hline
\end{tabular}

sPTPRG, soluble receptor-type tyrosine-protein phosphatase $\gamma$. 
Table 2:

Methods for isolation of exosomes or extracellular vesicles Yes, OK

\begin{tabular}{|c|c|c|c|}
\hline Isolation method (basic principal) & Method principal & Advantages & Limitations \\
\hline Ultracentrifugation & $\begin{array}{l}\text { Different sedimentation based } \\
\text { on particle density and size, can } \\
\text { be followed by sucrose gradient } \\
\text { and immunoaffinity methods } \\
\text { for better purity and isolation of } \\
\text { EV subtypes }\end{array}$ & $\begin{array}{l}\text { Most frequently used and } \\
\text { standardized method }\end{array}$ & $\begin{array}{l}\text { Low yield } \\
\text { Time consuming protocol } \\
\text { Expensive } \\
\text { Co-participation of protein } \\
\text { aggregate or viral lipoprotein } \\
\text { ab,108 }\end{array}$ \\
\hline Size exclusion techniques & $\begin{array}{l}\text { Serial filtration and } \\
\text { chromatography }\end{array}$ & $\begin{array}{l}\text { Inexpensive and not } \\
\text { technique sensitive }\end{array}$ & $\begin{array}{l}\text { Lack of efficient concentration of } \\
\text { EVs unless being used with } \\
\text { ultrafiltration concentrator } \\
\text { Risk of deflection and breakup of } \\
\text { large EVs due to the filtration } \\
\text { force }^{109}\end{array}$ \\
\hline Immune affinity isolation & $\begin{array}{l}\text { Antibody targeted toward } \\
\text { specific exosomes/EVs surface } \\
\text { marker }\end{array}$ & $\begin{array}{l}\text { Robust sensitivity and } \\
\text { specificity for isolation of } \\
\text { special subset of EVs }{ }^{110}\end{array}$ & $\begin{array}{l}\text { Expensive } \\
\text { Cross reactivity of antibody } \\
\text { Low yield }{ }^{111}\end{array}$ \\
\hline Microfluidic techniques & Isolating EVs in micro channels & $\begin{array}{l}\text { Low amount of initial EV- } \\
\text { containing fluid required } \\
\text { Possibility of combining } \\
\text { with immune affinity } \\
\text { methods }^{112}\end{array}$ & $\begin{array}{l}\text { Not validated } \\
\text { Low yield } \\
\text { Unproven efficiency and clinical } \\
\text { utility in comparative studies }\end{array}$ \\
\hline Polymeric Precipitation reagents & $\begin{array}{l}\text { Precipitation of exosomes or } \\
\text { EVs by means of dissolving } \\
\text { polymers }\end{array}$ & $\begin{array}{l}\text { Fast and efficient isolation } \\
\text { High yield of } \\
\text { exosomes/EVs and EV- } \\
\text { associated RNA } \\
\text { Efficient in clinical setting } \\
\text { Can be combined by } \\
\text { immune affinity isolation } \\
\text { methods to increase purity } \\
46,96\end{array}$ & $\begin{array}{l}\text { Risk of co-precipitation of proteins } \\
\text { and viruses, except when used in } \\
\text { combination with immune affinity } \\
\text { methods } 46\end{array}$ \\
\hline Porous structures $^{113}$ & $\begin{array}{l}\text { Trap small EVs through porous } \\
\text { microstructures }\end{array}$ & Fast & $\begin{array}{l}\text { Only suitable for isolation of small } \\
\text { EVs } \\
\text { No validation comparative study } \\
\text { available }^{112}\end{array}$ \\
\hline
\end{tabular}

EV, extracellular vesicle. 